\title{
Application of Mathematical Analysis Method in the Teaching of Automatic Control Theory
}

\author{
Xueyan Lin \\ Automation School \\ Beijing University of Posts and Telecommunications \\ Beijing, China \\ xylin@bupt.edu.cn
}

\begin{abstract}
In this paper, the method of root locus separation for complex automatic control system is discussed, to illustrate the importance of mathematical analysis methods in the design of automatic control systems. The mathematical method used to solve the separation points of root locus includes heuristic method, Newton residual theorem method, tail quadratic factor method and MATLAB method. This paper introduced the principle of these mathematical methods, analyzed and compared the solution process. The mathematical techniques used to obtain the separation point by heuristics, leaving the item not near the separation point, can make up for the shortcomings of the trial method with great blindness, reduce the number of calculations and increase its applicability. The Newton remainder theorem and the tail two factor method are tedious, but Newton's remainder theorem is more accurate. The Matlab method is faster and simpler.
\end{abstract}

Keywords-separation point of root trajectory, heuristic method, Newton residual theorem, tail second factor method, matlab

\section{INTRODUCTION}

The first mathematical courses of automatic control theory are advanced mathematics, complex function and integral transformation. The main content of the automatic control theory is to analyze and design the automatic control system. In detail, it uses time domain, frequency domain and root locus analysis method methods to analyze and design the performance (including stability, rapidity and accuracy) of mathematical models of automatic control system. The role of mathematical methods in the teaching knowledge points of the automatic control theory has been fully reflected.

Mathematical model of control system, whether it is differential equations, transfer functions, or frequency characteristics, is abstractions of the performance of the control system, and also the basis of studying control system. According to different mathematical analysis methods, different forms of mathematical models can be established. Different mathematical analysis methods can be adopted for different mathematical models. The differential equation is a time domain mathematical model of the control system. Time domain analysis studies what kind of time response the control system will produce under the given input. The mathematical analysis process first establish differential equations describing the system, obtains the solution of this equation to a given initial condition, and determine the stability and fast performance of the system with the time response. The advantage is more intuitive, easy to understand and accept. The drawback is the complexity of the solution, is difficult to find out the general law of the influence of differential equation coefficients and system element parameters on the system performance. Transfer function is a mathematical model of automatic control system in complex frequency domain. Its mathematical analysis process obtained the Laplace transform solution under certain input conditions, then the time-domain expression of the response with the inversion of the Laplace transform, and finally intuitive and accurate time response of the system outputs. The transfer function does not directly show the actual physical structure of the control system, but it can well reflect the system's own characteristics and control performance, and simplify the analysis process greatly. Frequency characteristic is a mathematical model of control system in frequency domain, the complex ratio between the steady-state output and the input of the system under sinusoidal signal, thus is a function of signal frequency. The frequency domain analysis includes amplitude frequency characteristics and phase frequency characteristics, for the complex characteristics of frequency characteristics (amplitude and phase). The advantage of the frequency method is that it is not necessary to calculate the entire process of output changes accurately, and the main performance index of the control system can be directly reflected by the structure, parameters and time of the system [1].

Since the position distribution of the closed-loop eigenvalue on the s-plane has a great influence on the performance of the closed-loop system, the response of the closed-loop system can be adjusted to achieve the expected performance by changing the position distribution of the closed-loop eigenvalue. Therefore it is very useful to master the influence of the system parameter on the position of the closed-loop characteristic root in the s-plane. Root trajectory method is one of the basic methods of analyzing and designing the control system in classical control theory. It is a graphic method. In 1948, E.R.Evans first proposed in "Graphical Analysis of Control Systems" [2] that when the open loop gain or other parameters change, all closed-loop poles corresponding to all values can be easily determined on the root locus. As the root trajectory graphically describes the distribution of the root of the system characteristic equation (the closed-loop pole of the system) on the s-plane. Therefore, 
it is very convenient to analyze the automatic control system with the root trajectory method, especially for high-order systems and multi-loop systems. It is more convenient to apply the root locus method than other methods, so it is widely used in engineering practice.

The root trajectory of the automatic control system is to follow the eight basic rules, in which the separation point and the intersection with the imaginary axis are two important rules which are computationally intensive and affect the correctness and accuracy of the root trajectory. The definition and characteristics of the separation point are clearly described in the textbook of the Automatic Control Principle [2-5], and it is proved that the separation point value $\mathrm{d}$ is the solution of the following equation:

$$
\sum_{j=1}^{m} \frac{1}{d+z_{j}}=\sum_{i=1}^{n} \frac{1}{d+p_{i}}
$$

Where $m$ is the open-loop zero points, $z_{j}$ is the value of each open-loop zero, $n$ is the number of open-loop poles, and $p_{i}$ is the value of each open-loop pole. For the vast majority of the control system, $n>m$, the equation of the separation point is unary $n$ equation. So solving the separation point is to solve the unary $\mathrm{n}$ equation. When the separation point equation is a quadratic equation $\mathrm{ad}^{2}+b d+c=0$, it is easily obtained by analytical method: $d_{1,2}=\frac{-b \pm \sqrt{b^{2}-4 a c}}{2 a}$. And then according to the characteristics of real axis between the two adjacent openloop poles must have the separation points, we can accurately determine the location of the separation point.

However, if the number of times of the separation point equation is higher than 2 , the solution is difficult, and the teaching method in textbook [2] about the separation point is described by the heuristic method to determine. The other textbooks are not discussing the process of solving the separation point, which the examples are simple and only by solving the quadratic equation can be determined. And this will make the students helpless when solve the root trajectory of the separation point in the complex control system. In the literature [7], the separation point of the root locus is solved by using the method of root weight and extreme value method, open-loop zero-pole method, Newton's residual theorem method and test method. However, due to the use of examples of the separation point equation is a simple binary quadratic equation, there is no practical significance. In addition, the significance of the method of using heavy root method, the extreme value method and the open loop zero pole method is that the meaning of the different expressions of the separation point equation is more important than the calculation method. In the literature [8], the heuristic method, root trajectory method and fast iteration method are introduced to solve the root of higher order algebraic equations.

In this paper, several common methods are used to solve the root trajectory separation point of a complex control system (shown in Fig. 1) by means of heuristics, Newton's remainder theorem, tail quadratic factor method and
MATLAB solution method, and the methods of these solutions are compared and analyzed.

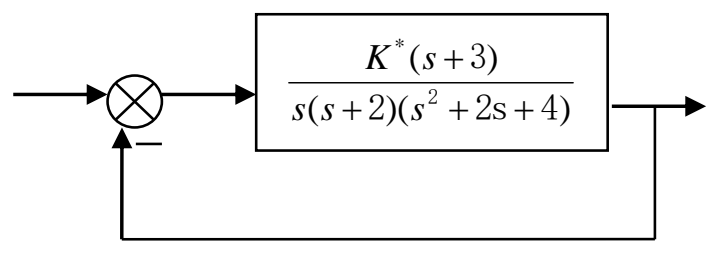

Fig. 1 Control system structure diagram

\section{SEPARATION POINT EQUATION}

The open-loop zero of the system is $z_{1}=-3$, the open-loop pole is $p_{1}=-0, p_{2}=-2, p_{3}=-1+j \sqrt{3}, p_{4}=-1-j \sqrt{3}$.

From equation (1) we can see that the separation point equation is:

$$
\frac{1}{d+3}=\frac{1}{d}+\frac{1}{d+2}+\frac{1}{\mathrm{~d}+1+j \sqrt{3}}+\frac{1}{\mathrm{~d}+1-j \sqrt{3}}
$$

Equation (3) can be available by formula (2):

$$
3 d^{4}+20 d^{3}+44 d^{2}+48 d+24=0
$$

The open - loop transfer function of the control system is $G(s) H(s)=\frac{K^{*} N(s)}{D(s)}$. Since the separation point is the repeated roots of the characteristic equation, the following two equations must be satisfied at the same time,

$$
\begin{aligned}
& \left\{\begin{array}{l}
1+G(s) H(s)=0 \Rightarrow K^{*} N(s)+D(s)=0 \\
{[1+G(s) H(s)]^{\prime}=0 \Rightarrow K^{*} N^{\prime}(s)+D^{\prime}(s)=0}
\end{array} \Rightarrow\right. \\
& N^{\prime}(s) D(s)-N(s) D^{\prime}(s)=0, \text { or } \frac{\mathrm{d}[G(s) H(s)]}{\mathrm{ds}}=0
\end{aligned}
$$

In this control system, $N(s)=(s+3)$,

$\mathrm{D}(\mathrm{s})=s(s+2)\left(s^{2}+2 \mathrm{~s}+4\right)$. It is also possible to obtain the separation point equation as shown in equation (3), which is the separation point equation obtained by the heavy root method.

$K^{*}=-\frac{D(s)}{\mathrm{N}(s)}$ Can be drawn by the characteristic equation $1+G(s) H(s)=0$. We can find the extreme value of $\mathrm{K}^{*}$. $\frac{d K^{*}}{d s}=-\frac{D^{\prime}(s) N(s)-D(s) N^{\prime}(s)}{\mathrm{N}^{2}(s)}=0$. The separation point equation of equation (3) can also be obtained.

This is unary 4 equation that can't be solved by a simple arithmetic method. In this paper, the complex control system refers to the root trajectory separation point equation is more than 2. This article describes the following several methods of solution, and make a comparative analysis. 


\section{METHOD FOR OBTAINING SEPARATION POINT}

\section{A. Heuristic method}

The separation point $\mathrm{d}$ should be between the two consecutive open-loop poles $[-2,0]$ and open-loop zeros $(-\infty$ $-3]$. Therefore, the median $(-1)$ of the interval $[-2,0]$ is taken as the first test value. It is substituted into the formula (2), left $=$ 0.5 , right $=0$, the difference is 0.5 . The median $(-1.5)$ of the interval $[-2,-1]$ is taken as the second test and it is substituted into the formula (2), the left $=0.67$, right $=1.02$, the difference is 0.35 . After 5 tests according to the value of each test for the median range, then $\mathrm{d}=-1.38$, left $=0.62$, right $=0.65$, the difference is 0.03 . Fully meet the requirements.

To avoid blindness and reduce the number of iterations, use the following test techniques. The trick is leaving one without being brought into. $\mathrm{d}$ which is not being brought into should be closer to the separation point, it is not taken at random. In this case, the separation point is between $[-2,0]$, which takes the $1 /$ $(d+2)$ or the $1 / d$, the invariant, and then the others. Here take 1 $/(d+2)$ unchanged, and do the following test.

The first step is to make $d=-1$ then

$$
\frac{1}{d+2}=\frac{1}{d+3}-\frac{1}{d}-\frac{2 \mathrm{~d}+2}{d^{2}+2 \mathrm{~d}+4}=1.5 \text {. }
$$

So the solution is: $\mathrm{d}=-1.33$, compared to the known result, the error of $d_{1}=-1.38$ is $3.6 \%$. Meet the requirements. The advantage is that it reduces the number of attempts.

With this technique, another separation point can be obtained in only 2 steps. Since the $\mathrm{d}_{2}$ separation point is between $(-\infty,-3]$. Take $1 /(d+3)$ unchanged, so $d_{2}=-4$ and take into the formula (2) then $\frac{1}{d+3}=1.25 \Rightarrow \mathrm{d}=-3.8$. Compared to the known result, the error of $d_{2}=-3.7$ is $2.6 \%$.

\section{B. Newton remainder theorem}

The Newton Remainder Theorem is a method proposed by Newton in the 17th century to solve the equation $\mathrm{f}(\mathrm{x})=0$ approximation. Given that the range of $\mathrm{f}(\mathrm{x})=0$, any $\mathrm{x}_{0}$ is in the range as its initial approximation. $\left(\mathrm{x}-\mathrm{x}_{0}\right)$ is divided by $\mathrm{f}(\mathrm{x})$ and the quotient is denoted as $\mathrm{Q}_{1}(\mathrm{x})$, the remainder is denoted by $R_{1}$. And then $\left(x-x_{0}\right)$ is divided by $Q_{1}(x)$, the remainder is $R_{2}$ Thus, $x_{1}=x_{0}-R_{1} / R_{2}$ is obtained. The resulting $x_{1}$ is an approximation of the solution better than $\mathrm{x}_{0}$ and has high precision requirements. Repeat the above steps, $\left(\mathrm{x}-\mathrm{x}_{1}\right)$ is divided by $\mathrm{f}(\mathrm{x})$ to get the quotient as $\mathrm{Q}_{2}(\mathrm{x})$, the remainder is $\mathrm{R}_{3}$. And then $\left(\mathrm{x}-\mathrm{x}_{1}\right)$ is divided by $\mathrm{Q}_{2}(\mathrm{x})$, the remainder is taken as $\mathrm{R}_{4}$. Thus, $x_{2}=x_{1}+R_{3} / R_{4}$ is obtained. The resulting $\mathrm{x}_{2}$ is an approximation of the solution better than $x_{1}$. For more repetitions, a more accurate approximation is obtained. Automatic Control Principle published by South China University of Technology [9] is using this method to solve the root trajectory of the separation point.

For the equation of separation of equation (3), the first calculation takes $\mathrm{d}_{0}=-1$, that is, $(\mathrm{d}+1)$ is divided by equation (3), and the quotient is $Q_{1}(d)=3 d^{3}+17 d^{2}+27 d+21$, the remainder is $R_{1}=3$. Then $(\mathrm{d}+1)$ is divided by $Q_{1}(d)$, and the quotient is $Q_{2}(d)=3 d^{2}+14 d+13$, the remainder is $R_{2}=8$.
So, $d_{1}=d_{0}-\frac{R_{1}}{R_{2}}=-1-\frac{3}{8}=-1.38$.It has been consistent with the answer.

Calculate another separation point, $(\mathrm{d}+4)$ is divided by equation (3), and the quotient is $Q_{1}(d)=3 d^{3}+8 d^{2}+12 d$, the remainder is $R_{1}=0$. So the separation point is -4 .

\section{Tail quadratic factor method}

The tail quadratic factor method is a kind of splitting factor method [10, 11]. Its concrete solving process for high order equation () $n_{f \neq x}{ }_{1} *^{n-1} a x \frac{1}{2}^{n-2} a x+{ }_{n-2} a^{2} x+a_{n} x_{1} x+a_{n}=0$ is shown as follows: (1) Take the tail quadratic factor $x^{2}+\frac{a_{n-1}}{a_{n-2}} x^{1}+\frac{a_{n}}{a_{n-2}}$ divided by $\mathrm{f}(\mathrm{x})$, until the emergence of the formula for the second factor $\mathrm{b}_{n-2} x^{2}+b_{n-1} x^{1}+b_{n}$. (2) Take $x^{2}+\frac{\mathrm{b}_{n-1}}{b_{n-2}} x^{1}+\frac{b_{n}}{b_{n-2}}$ divided by the left side of the equation, until the residual equation is the second factor $\mathrm{c}_{n-2} x^{2}+c_{n-1} x^{1}+c_{n}$. (3) Repeat the above work until the last resulting surplus and the last division are approximately equal. Automatic Control Principle of Hefei University of Technology and Control Engineering Foundation of Tsinghua University Press both introduced the cleavage method to solve the separation point.

For the separation point of equation (3), the ratio of the coefficients between items is:

$$
\begin{aligned}
& \mathrm{S}_{1}=\frac{20}{3}=6.67, \mathrm{~S}_{2}=\frac{44}{20}=2.2, \\
& \mathrm{~S}_{3}=\frac{48}{44}=1.09, \mathrm{~S}_{4}=\frac{24}{48}=0.5,
\end{aligned}
$$

The condition that satisfies the use of the tail quadratic factor method "has two very close to the smaller $S_{n}$ value". The calculation process is shown in Table 1.

TABLE I ITERATIVE COMPUTING PROCESS OF TAIL FACTOR

\begin{tabular}{|c|l|l|c|}
\hline $\begin{array}{l}\text { Number of } \\
\text { iterations }\end{array}$ & Tail factor & Quotient & Remainder \\
\hline 1 & $d^{2}+1.1 d+0.54$ & $3 d^{2}+16.7 d+24$ & $12.6 d+11$ \\
\hline 2 & $d^{2}+1.62 d+1$ & $3 d^{2}+15.1 d+16.5$ & $6.2 d+7.53$ \\
\hline 3 & $d^{2}+2 d+1.46$ & $3 d^{2}+14 d+11.6$ & $4.3 d+7$ \\
\hline 4 & $d^{2}+2.37 d+2.06$ & $3 d^{2}+12.9 d+7.3$ & $4.2 d+9$ \\
\hline 5 & $d^{2}+2.95 d+3.3$ & $3 d^{2}+11.2 d+1.2$ & $5 d+20$ \\
\hline
\end{tabular}

So the separation point equation can be decomposed into two quadratic factor multiplying:

$$
\begin{aligned}
& 3 d^{4}+20 d^{3}+44 d^{2}+48 d+24 \\
& =\left(d^{2}+2 d+1.46\right)\left(3 d^{2}+14 d+11.6\right)=0
\end{aligned}
$$

Then, $d_{1,2}=-1 \pm j 0.68($ abandoned $), \quad d_{3}=-1.08, d_{4}=-3.6$

The separation point $d_{3}$ and the known answer -1.38 have a large error of $21.7 \%$. The separation point $\mathrm{d}_{4}$ and the known answer -3.7 have a small error of $2.7 \%$. 


\section{Application of MATLAB simulation calculation}

Using the polynomial root function roots () of MATLAB, it is easy to get the roots of polynomials. The MATLAB text statement is: $\mathrm{P}=[3,20,44,48,24]$; $\mathrm{d}=\operatorname{roots}(\mathrm{P})$.

The result is: $\mathrm{d}=-3.7310 ;-1.3662 ;-0.7847+0.9766 \mathrm{i}$ (abandoned); -0.7847- 0.9766i (abandoned).

\section{COMPARISON OF SEPARATION POINTS}

The method of finding the separation point is compared between the error, the number of calculations, and the characteristics, as shown in Table 2 .

TABLE II COMPARISON OF SEPARATION POINTS

\begin{tabular}{|c|c|c|c|c|}
\hline name & Results & Error & $\begin{array}{l}\text { Number } \\
\text { of } \\
\text { calculate } \\
\text { times } \\
\end{array}$ & Key description \\
\hline $\begin{array}{l}\text { Heuristic } \\
\text { techniques }\end{array}$ & $\begin{array}{l}-1.33 \\
-3.8\end{array}$ & $\begin{array}{l}3.6 \% \\
2.6 \%\end{array}$ & 2 & $\begin{array}{l}\text { Prefetching separation } \\
\text { point; } \\
\text { The point away from } \\
\text { separation point is not } \\
\text { brought into; Multiple } \\
\text { separation points are } \\
\text { calculated separately. }\end{array}$ \\
\hline $\begin{array}{l}\text { Newton } \\
\text { remainder } \\
\text { theorem }\end{array}$ & $\begin{array}{c}-1.38 \\
-4\end{array}$ & $\begin{array}{c}0.0 \% \\
8 \%\end{array}$ & $2 ; 1$ & $\begin{array}{c}\text { Prefetching separation } \\
\text { point; Factor in long } \\
\text { division. }\end{array}$ \\
\hline $\begin{array}{l}\text { Tail } \\
\text { quadratic } \\
\text { factor } \\
\text { method }\end{array}$ & $\begin{array}{l}-1.08 \\
-3.6\end{array}$ & $\begin{array}{c}21.7 \% \\
2.7 \%\end{array}$ & 3 & $\begin{array}{l}\text { Factor in long division; } \\
\text { The coefficient of the tail } \\
\text { quadratic factor is } 1 ; \\
\text { Find two separation } \\
\text { points at the same time. }\end{array}$ \\
\hline $\begin{array}{l}\text { MATLAB } \\
\text { simulation } \\
\text { method }\end{array}$ & $\begin{array}{l}-1.37 \\
-3.7\end{array}$ & $0.0 \%$ & 2 & $\begin{array}{l}\text { Use the proper function } \\
\text { of MATLAB software; }\end{array}$ \\
\hline
\end{tabular}

\section{CONCLUSION}

The three analysis methods in automatic control theory are the embodiment and application of mathematical thought. Having a good knowledge of mathematical theory, and a certain level of mathematical thinking, is the basis and premise of learning automatic control theory and other engineering theories.
The following four methods can be used to find the separation point of complex system: heuristic technique, Newton residual theorem, tail quadratic factor method and MATLAB simulation method.

With the heuristic method to find the separation point, "leaving the point that near the separation point does not bring into" the calculation technique, can make up for the trial method with a large blindness of the shortcomings, reducing the number of calculations, increased its applicability.

With the Newton's remainder theorem and the tail quadratic factor method to find the points, they need to adopt the calculation process of the formula, and the calculation is more complicated. The Newton's Remainder Theorem requires prefetching separation point, but the result is more accurate than the tail quadratic factor method.

These methods have good convergence in finding the separation point. We can adopt a method which we are interested when using, and we can find the separation point quickly and accurately.

\section{REFERENCES}

[1] Yuan Yuan. Application of mathematical model and mathematica analysis method in automatic control theory. Vocational \& Technical Education Forum. 2003(10)

[2] Shou-Song Hu. Automatic Control Principle (Sixth Edition) [M] Beijing: Science Press, 2013.

[3] Jing-Xin Dong, Chang-De. Control Engineering Foundation (second edition) [M]. Tsinghua University Press, 2003.

[4] Yan-Shuo Ren. Automatic Control Principle [M]. Mechanical Industry Press, 2007.

[5] Hua Meng. Automatic Control Principle [M]. Mechanical Industry Press 2007.

[6] Jian-Hui Wang, Shu-Sheng Gu. Automatic Control Principle [M].Tsinghua University Press, 2007.

[7] Yang Wang. Root trajectory of the separation point (or rendezvous point) of the several methods. Xianning College Journal 24(6), 2004.

[8] Xue-Wu Wang. Root trajectory separation point calculation method Changchun Institute of Optics and Fine Mechanics. 13(3), 1990.

[9] Guo-Shen Gao, Wen-Jie Yu. Automatic Control Principle (Forth Edition) [M]. South China University of Technology Press, 2013.

[10] Shi-E Lin, On the Application of the Phenomenon Method to Solve the Root Problem of Higher Order Characteristic Equation. Progress in Mathematics 1963 (3): 3-13.

[11] Jing-Xin Dong, Chang-De Zhao, Control Engineering Foundation. Tsinghua University Press, 1992. 